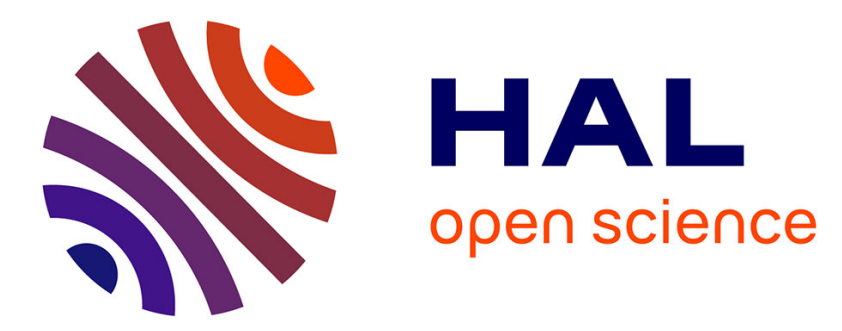

\title{
A search for manifestation of two types of collective excitations in dynamic structure of a liquid metal: Ab initio study of collective excitations in liquid $\mathrm{Na}$
}

Taras Bryk, J. -F. Wax

\section{- To cite this version:}

Taras Bryk, J. -F. Wax. A search for manifestation of two types of collective excitations in dynamic structure of a liquid metal: Ab initio study of collective excitations in liquid Na. Journal of Chemical Physics, 2016, 144 (19), pp.194501. 10.1063/1.4948709 . hal-01513807

\section{HAL Id: hal-01513807 \\ https://hal.univ-lorraine.fr/hal-01513807}

Submitted on 9 May 2017

HAL is a multi-disciplinary open access archive for the deposit and dissemination of scientific research documents, whether they are published or not. The documents may come from teaching and research institutions in France or abroad, or from public or private research centers.
L'archive ouverte pluridisciplinaire HAL, est destinée au dépôt et à la diffusion de documents scientifiques de niveau recherche, publiés ou non, émanant des établissements d'enseignement et de recherche français ou étrangers, des laboratoires publics ou privés. 


\section{A search for manifestation of two types of collective excitations in dynamic structure of a liquid metal: Ab initio study of collective excitations in liquid $\mathrm{Na}$}

Taras Bryk and J.-F. Wax

Citation: The Journal of Chemical Physics 144, 194501 (2016); doi: 10.1063/1.4948709

View online: http://dx.doi.org/10.1063/1.4948709

View Table of Contents: http://aip.scitation.org/toc/jcp/144/19

Published by the American Institute of Physics

\section{Articles you may be interested in}

Erratum: "A search for manifestation of two types of collective excitations in dynamic structure of a liquid metal: Ab initio study of collective excitations in liquid Na" [J. Chem. Phys. 144, 194501 (2016)]

The Journal of Chemical Physics 144, 219903219903 (2016); 10.1063/1.4953241

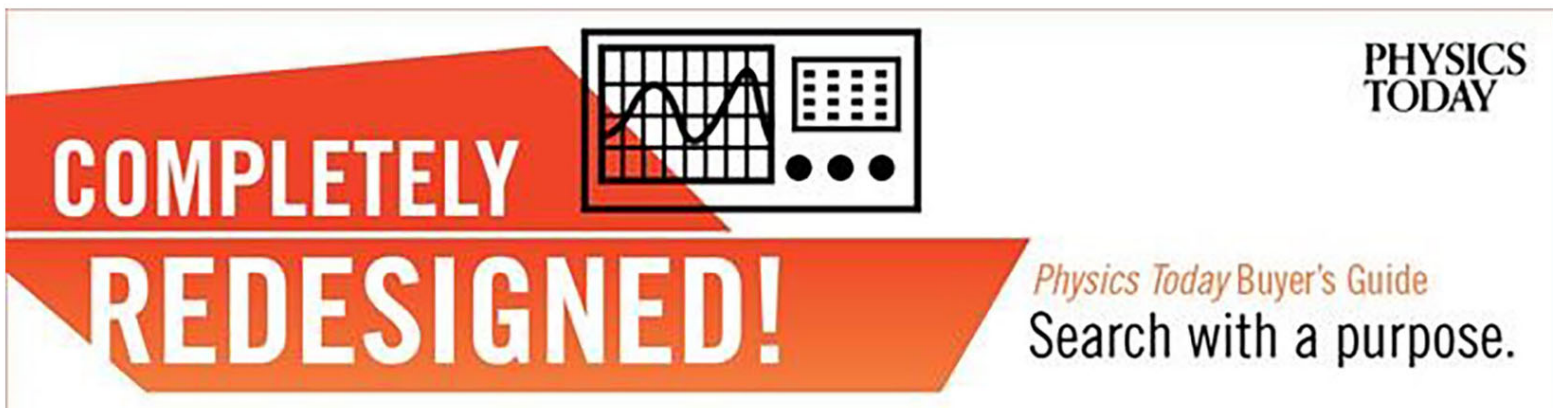




\title{
A search for manifestation of two types of collective excitations in dynamic structure of a liquid metal: $A b$ initio study of collective excitations in liquid $\mathrm{Na}$
}

\author{
Taras Bryk ${ }^{1}$ and J.-F. Wax ${ }^{2}$ \\ ${ }^{1}$ Institute for Condensed Matter Physics, National Academy of Sciences of Ukraine, 1 Svientsitskii Street, \\ UA-79011 Lviv, Ukraine and Institute of Applied Mathematics and Fundamental Sciences, Lviv Polytechnic \\ National University, UA-79013 Lviv, Ukraine \\ ${ }^{2}$ Université de Lorraine, LCP-A2MC, EA4632, 1, boulevard Arago, 57078 Metz Cedex 3, France
}

(Received 9 March 2016; accepted 25 April 2016; published online 16 May 2016)

\begin{abstract}
Using a combination of $a b$ initio molecular dynamics and several fit models for dynamic structure of liquid metals, we explore an issue of possible manifestation of non-acoustic collective excitations in longitudinal dynamics having liquid $\mathrm{Na}$ as a case study. A model with two damped harmonic oscillators (DHOs) in time domain is used for analysis of the density-density time correlation functions. Another similar model with two propagating contributions and three lowest exact sum rules is considered, as well as an extended hydrodynamic model known as thermo-viscoelastic one which permits two types of propagating modes outside the hydrodynamic region to be used for comparison with ab initio obtained time correlation functions and calculations of dispersions of collective excitations. Our results do not support recent suggestions that, even in simple liquid metals, non-hydrodynamics transverse excitations contribute to the longitudinal collective dynamics and can be detected as a DHO-like spectral shape at their transverse frequency. We found that the thermoviscoelastic dynamic model permits perfect description of the density-density and current-current time correlation functions of the liquid $\mathrm{Na}$ in a wide range of wave numbers, which implies that the origin of the non-hydrodynamic collective excitations contributing to longitudinal dynamics can be short-wavelength heat waves. Published by AIP Publishing. [http://dx.doi.org/10.1063/1.4948709]
\end{abstract}

\section{INTRODUCTION}

Collective dynamics in liquids being one of the fascinating problems of condensed matter physics is well understood only on macroscopic length and time scales, where Brillouin light scattering and hydrodynamic theory explain in nice agreement the observed three-peak shape of the low-frequency spectrum of the scattered light. ${ }^{1,2}$ On atomic-scale resolution, the microscopic collective dynamics can be probed by inelastic Xray scattering (IXS) or inelastic neutron scattering (INS), and the experimental spectra need to be properly analyzed in order to estimate the dispersion of collective excitations outside the hydrodynamic regime as well as to obtain relevant information about many possible non-hydrodynamic relaxation processes contributing to collective dynamics. ${ }^{3}$ A very specific feature of collective dynamics in liquids is the role of thermal fluctuations, because macroscopic propagation of sound which is the adiabatic one causes different temperatures for the compression and decompression regions in the sound wave. The permanently fluctuating temperature field in liquids causes diffusivity of local temperature (thermal diffusivity) that makes thermal relaxation the leading relaxation process on macroscopic scales in liquids. On molecular spatial scale, non-hydrodynamic propagating processes can contribute to thermal fluxes ${ }^{4}$ too. These features of temperature fluctuations in liquids must be taken into account in analysis of collective dynamics outside the hydrodynamic regime.
One of the simplest non-hydrodynamic processes is the structural relaxation, which contributes to the central peak of dynamic structure factor $S(k, \omega)(k$ and $\omega$ are wave number and frequency, respectively) outside the hydrodynamic region ${ }^{5,6}$ and which corresponds to structural rearrangement of atoms in liquid. Since on macroscopic scale (for which no atomistic structure is resolved) the collective dynamics in liquids is constrained by the local conservation laws which form a set of hydrodynamic equations for liquid, the only relaxing mode contributing in this range to the central peak of $S(k, \omega)$ is the one connected with diffusivity of local temperature (thermodiffusive mode). The hydrodynamic theory gives evidence that the contribution from the hydrodynamic thermodiffusive mode to $S(k, \omega)$ is finite and equal to $S(k=0)(\gamma-1) / \gamma$, where $S(k)$ is the static structure factor tending to its nonzero value defined by the isothermal compressibility and $\gamma$ is the ratio of specific heats. In contrast, the contribution from non-hydrodynamic structural relaxation tends to be zero as $\sim k^{4}$ in the long-wavelength limit. ${ }^{5}$ However, outside the hydrodynamic regime, the structural relaxation strongly increases its contribution to $S(k, \omega)$ and in the region of the main peak of static structure factor $S(k)$ almost completely defines the central peak of dynamic structure factor. ${ }^{6}$

The other collective non-hydrodynamic modes such as shear or heat waves $^{7}$ do not exist on macroscopic scales; however, they can propagate with relatively large damping on nanoscales in liquids. The contributions from heat waves 
to dynamic structure factors $S(k, \omega)$ in supercritical Ar were studied recently ${ }^{8}$ in connection with their possible effect on the positive sound dispersion in gas-like fluids. However, the contributions from shear waves to $S(k, \omega)$ were not expected in simple non-molecular liquids because the cross-correlations between the longitudinal (L) and transverse ( $\mathrm{T}$ ) components of currents and of stress tensor are zero due to symmetry rules. Hydrodynamic equations are decoupled for $\mathrm{L}$ and $\mathrm{T}$ components. So far there is no elaborated generalized hydrodynamic theory for pure liquids with coupled $\mathrm{L}$ and $\mathrm{T}$ dynamics outside the hydrodynamic regime, which would be supported by MD simulations. In case of molecular liquids like water, the manifestation of the transverse excitations in longitudinal dynamics was observed in MD simulations ${ }^{9}$ and scattering experiments. ${ }^{10}$ Nevertheless, although having a clear hint of crucial role of the rotational degrees of freedom in the mixing of $\mathrm{L}$ and $\mathrm{T}$ dynamics, there is no robust simulation-validated generalized hydrodynamic theory in the case of molecular liquids too.

Recently there appeared reports on experimental IXS studies in which the observation of contributions from Texcitations to dynamic structure factor of pure liquid $\mathrm{Ga}^{11,12}$ and $\mathrm{Sn}^{13}$ was claimed. Similar findings were reported from a fit analysis of IXS experiments for liquid $\mathrm{Na}$ at temperature $393 \mathrm{~K},{ }^{14} \mathrm{Cu}$ at $1423 \mathrm{~K},{ }^{15} \mathrm{Fe}$ at $1823 \mathrm{~K},{ }^{15}$ and $\mathrm{Zn}$ at $713 \mathrm{~K},{ }^{15}$ and another result from INS experiments on liquid $\mathrm{Zn}$ at $773 \mathrm{~K}$ was reported in Ref. 16.

On the simulation side, there were just a few studies in which were observed more than one peak (or additional shoulder) of the longitudinal and transverse current spectral functions $C^{L / T}(k, \omega)$, attributed to a possible coupling between the $\mathrm{L}$ and $\mathrm{T}$ collective excitations on the molecular length scale. In Refs. 17 and 13, the case of liquid Sn was studied, and $a b$ initio simulations performed for three temperatures of $573 \mathrm{~K}, 873 \mathrm{~K}$, and $1273 \mathrm{~K}$ gave evidence of several propagating modes contributing to the shape of $C^{L / T}(k, \omega)$. In the case of liquid $\mathrm{Li}$ at temperature $1000 \mathrm{~K}$ and ambient pressure, ${ }^{18}$ it was observed in $a b$ initio MD simulations just a standard one-peak behaviour of $C^{L / T}(k, \omega)$. However, the applied pressure up to $126 \mathrm{GPa}$ showed an emergence of a weak high-frequency maximum on the transverse current spectral function, which was resolved for large wave numbers in the longitudinal $C^{L}(k, \omega)$ too. Moreover, it was shown in Ref. 18 that the regular viscoelastic theory for transverse dynamics with only one type of collective excitations (shear waves) was unable to recover the transverse current autocorrelation function at high pressures. Only an extended hydrodynamic approach with two types of coupled collective excitations enabled to recover the $a b$ initio molecular dynamics (AIMD)-derived transverse current autocorrelation function that was the strong evidence of two types of collective excitations contributing to the transverse dynamics of liquid $\mathrm{Li}$ at high pressures. We can also mention an $a b$ initio molecular dynamics study of liquid $\mathrm{Fe}^{19}$ in which weak shoulders in dynamic structure factor $S(k, \omega)$ in the region of wave numbers $0.93-1.31 \AA^{-1}$ were interpreted as due to contribution coming from T-excitations, although neither theoretical models nor a fit was used for the identification of the transverse origin of these weak shoulders.
The analysis of the experimental IXS and INS intensities with a purpose to obtain some signal from a non-acoustic collective excitation is pretty simple and so far it was not based on any robust theoretical approach. Furthermore, it was not clear from the fit analysis with two damped harmonic oscillators (DHOs) or Gaussians ${ }^{15}$ what level of exact sum rules was satisfied by the fit ansatz. The only criterion which was used in the analysis of the scattering intensities to ascribe the obtained non-acoustic DHO-frequency to the transverse collective excitations was a more-or-less correspondence of these frequencies in some (sometimes very narrow as in Ref. 11) range of wave numbers to dispersions of transverse excitations obtained from $a b$ initio simulations.

One of the most logical and simple checks of the suggested manifestation of T-excitations in the dynamic structure factor $S(k, \omega)$ of pure liquid metals is the $a b$ initio simulation of their collective dynamics and verification of the different dynamic models used for estimation of the contributions coming supposedly from T-modes to $S(k, \omega)$. The $a b$ initio simulations are free of parametrized effective pair interatomic interactions and allow calculations of microscopic forces acting on atomistic particles via instantaneous distribution of electron density, which is calculated from Kohn-Sham wave functions. The advantage of an analysis of collective dynamics from $a b$ initio simulations is in possibility to focus on density-density time correlation functions, which can be calculated with high precision and which are directly connected with the dynamic structure factor via time-Fourier transform. Another very strong argument is in simplicity to apply in the fitting of the time correlation functions the exact sum rules which define their short-time behaviour.

Hence, the aim of this study is to perform $a b$ initio simulations of liquid $\mathrm{Na}$ at the experimental temperature, calculate well-converged density-density time correlation functions, and apply for their analysis several models of collective dynamics including the ones with two (supposedly corresponding to $\mathrm{L}$ and $\mathrm{T}$ excitations) DHOs with and without applied sum rules, as well as a generalized hydrodynamic model of longitudinal dynamics. The remaining paper has the following structure: in Sec. II, we give information on the $a b$ initio simulations and fitting models used in this study. Section III contains the analysis of the simulation-derived density-density time correlation functions and discussion on the obtained results. The conclusions of this study are given in Sec. IV.

\section{METHODOLOGY}

$A b$ initio simulations were performed for liquid $\mathrm{Na}$ at temperature $393 \mathrm{~K}$ using a system of 300 atomistic particles interacting with the electronic subsystem via PAW potentials (electron-ion interaction preserving the nodal structure of valence electron wave functions). ${ }^{23,24}$ The density of the simulated system was estimated from adjusting the ambient pressure of 1 atm and resulted in the number density of $0.0276 \AA^{-3}$. Since liquid $\mathrm{Na}$ at ambient conditions is a simple metal, we made use of the local density approximation (LDA) 
for exchange-correlation functional. Although it would be interesting to explore an effect of different approximations of the exchange-correlation potential on transport properties and collective excitations (as it was reported recently for the case of diffusivity and viscosity in liquid $\mathrm{Al}^{25}$ and $\mathrm{Cu}^{26}$ ), we restricted ourselves just with the simplest LDA having the primary aim to study the possibility of manifestation of non-hydrodynamic excitations in dynamic structure factors. Furthermore, in Ref. 26, it was shown that, namely, LDA based results were in close agreement with experimental data for liquid $\mathrm{Cu}$ for both the self-diffusion coefficient and the viscosity over the temperature range investigated.

The electron density was calculated using just a single $\Gamma$ point in the Brillouin zone that is well justified by quite large size of model system. Upon an equilibration over 4000 time steps with $\Delta t=3 \mathrm{fs}$, we performed the equilibrium production run over 20000 steps that allowed us to obtain well converged static averages and time correlation functions. All the wavenumber dependent quantities were averaged over all possible directions of wave vectors corresponding to the same absolute value. The smallest wave number was $0.284 \AA^{-1}$.

A fit ansatz containing two DHOs (supposedly of L- and T-origin) used in Ref. 14 consisted for inelastic contribution of two intensities

$$
I_{D H O}(k, \omega)=\frac{4 \Gamma(k) \Omega^{2}}{\left(\omega^{2}-\Omega^{2}(k)\right)^{2}+4 \omega^{2}(k) \Gamma^{2}(k)} .
$$

Each DHO-intensity is equivalent to the standard sum ${ }^{20}$ of side Lorentzians centered at the frequencies $\pm \omega_{s}(k)$ and nonLorentzian contributions which are very similar to the form that follows from hydrodynamic theory for acoustic modes with an account for a few lowest sum rules ${ }^{21}$

$$
\begin{aligned}
I_{D H O}(k, \omega)= & \frac{\Gamma(k)+\left(\omega+\omega_{s}(k)\right) \tan \phi}{\left(\omega+\omega_{s}(k)\right)^{2}+\Gamma^{2}(k)} \\
& +\frac{\Gamma(k)-\left(\omega-\omega_{s}(k)\right) \tan \phi}{\left(\omega-\omega_{s}(k)\right)^{2}+\Gamma^{2}(k)},
\end{aligned}
$$

where

$$
\tan \phi=\Gamma(k) / \omega_{s}(k) .
$$

Note that the frequencies $\omega_{s}(k)$ and $\Omega(k)$ are not the same because

$$
\omega_{s}^{2}(k)=\Omega^{2}(k)-\Gamma^{2}(k) .
$$

Outside the hydrodynamic region, the damping of collective excitations increases strongly causing a difference between the frequencies $\Omega$ and $\omega_{s}$. Note that $\Omega$ is exactly the maximum frequency for the DHO intensity (1) multiplied by $\omega^{2}$, i.e., it should correspond to the peak position of the current spectral function in case of just a single propagating contribution to $C^{L}(k, \omega)$. As it was mentioned in Ref. 22, " $\Omega$ is the physically meaningful frequency, while $\omega_{s}$ appears to be close to the visible maximum of the signal." In time domain, the single DHO (1) corresponds to a damped harmonic oscillation

$$
F_{D H O}(k, t)=B \cos \left(\omega_{s}(k) t-\phi\right) e^{-\Gamma(k) t},
$$

which has the first time derivative at origin equal to zero because of relation (3).

In our study, the density-density time correlation functions calculated from AIMD were analyzed by several different models as follows:

(i) Model M1 which is the time-dependent form of the two DHOs (2) and one central Lorentzian

$$
\frac{F_{n n}^{M 1}(k, t)}{F_{n n}(k, t=0)}=A_{n n}(k) e^{-d(k) t}+\sum_{\alpha=1}^{2} B_{n n}^{\alpha}(k)\left[\cos \left(\omega_{\alpha}(k) t\right)+\frac{\sigma_{\alpha}(k)}{\omega_{\alpha}(k)} \sin \left(\omega_{\alpha}(k) t\right)\right] e^{-\sigma_{\alpha}(k) t} .
$$

The lowest sum rule $F_{n n}^{M 1}(k, t=0)=S(k)$ results in the relation

$$
A_{n n}(k)=1-B_{n n}^{1}(k)-B_{n n}^{2}(k),
$$

hence reducing the number of free parameters to seven: $d(k)$, $B_{n n}^{\alpha}(k), \omega_{\alpha}(k), \sigma_{\alpha}(k), \alpha=1,2$. (ii) Model M2 which is quite similar time-dependent form of the two symmetric (Lorentzian) and asymmetric (nonLorentzian) contributions to side peaks and one central Lorentzian, but with the first three (constrained short-time behaviour of $F_{n n}^{M 2}(k, t)$ with additional first and second time derivatives at the origin) fulfilled sum rules,

$$
\frac{F_{n n}^{M 2}(k, t)}{F_{n n}(k, t=0)}=A_{n n}(k) e^{-d(k) t}+\sum_{\alpha=1}^{2}\left[B_{n n}^{\alpha}(k) \cos \left(\omega_{\alpha}(k) t\right)+D_{n n}^{\alpha}(k) \omega_{\alpha}(k) \sin \left(\omega_{\alpha}(k) t\right)\right] e^{-\sigma_{\alpha}(k) t} .
$$

This ansatz with three constraints for the sum rules allows to have the same seven free parameters: $d(k), B_{n n}^{\alpha}(k), \omega_{\alpha}(k)$, $\sigma_{\alpha}(k), \alpha=1,2$. (iii) The generalized hydrodynamic 5-variable model, known as the thermo-viscoelastic one, which is applied for solving the generalized Langevin equation in a matrix form constructed 
on a set of five dynamic variables

$\mathbf{A}^{(5)}(k, t)=\left\{n(k, t), J^{L}(k, t), e(k, t), \dot{J}^{L}(k, t), \dot{e}(k, t)\right\}$,

where $n(k, t), J^{L}(k, t)$, and $e(k, t)$ are the Fourier-components of hydrodynamic variables of particle density, density of longitudinal component of mass current, and energy density, respectively. The extended (non-hydrodynamic) variables are represented via the first time derivatives of longitudinal current and energy densities and are marked as dotted ones. The $5 \times 5$ generalized hydrodynamic matrix $\mathbf{T}(k)$ is expressed in frames of the approach of generalized collective modes $(\mathrm{GCMs})^{27}$ in the following way:

$$
\mathbf{T}(k)=\mathbf{F}(k, t=0) \tilde{\mathbf{F}}^{-1}(k, z=0)
$$

via the $5 \times 5$ matrices of static correlation functions $\mathbf{F}(k, t=0)$ and of Laplace-transformed time correlation functions in Markovian approximation $\tilde{\mathbf{F}}(k, z=0)$. The matrix elements of $\mathbf{F}(k, t=0)$ and $\tilde{\mathbf{F}}(k, z=0)$ for the basis set (7) were given in Ref. 28. The matrix elements for correlators between $n(k, t), J^{L}(k, t)$, and $\dot{J}^{L}(k, t)$ can be easily calculated directly in AIMD, while extremely time-consuming calculations of matrix elements involving energy density and its time derivative were replaced by free parameters obtained from the fit. Hence, four matrix elements (see Ref. 28) $f_{n e}(k), f_{e e}(k)$, $f_{\dot{J} e}(k), f_{\dot{e} \dot{e}}$, and two correlation times $\tau_{n e}(k), \tau_{e e}(k)$ were treated as fitting parameters. The fitting can be performed simultaneously for two AIMD-derived functions: densitydensity and longitudinal current-current ones. Note that in this GCM scheme the mode weights $A_{i i}^{\alpha}(k), B_{i i}^{\alpha}(k)$, $D_{i i}^{\alpha}(k)$ as well as inverse relaxation times of relaxing modes, real eigenvalues of $\mathbf{T}(k), d_{i}(k)$, frequencies $\omega_{i}(k)$ and damping $\sigma_{i}(k)$ of propagating eigenmodes, complexconjugated pairs of eigenvalues of $\mathbf{T}(k), \sigma_{\alpha}(k) \pm i \omega_{\alpha}(k)$ are not fitting parameters. They are estimated from the eigenvalues and corresponding eigenvectors of $\mathbf{T}(k)$, which contains the abovementioned six fitting parameters in its matrix elements. ${ }^{27,28}$ The GCM expressions for the replicas of time correlation functions within the thermo-viscoelastic model (7) $\operatorname{read}^{29}$

$$
\frac{F_{i i}^{G C M}(k, t)}{F_{i i}(k, t=0)}=\sum_{\alpha=1}^{3(1)} A_{i i}^{\alpha}(k) e^{-d_{\alpha}(k) t}+\sum_{\alpha=1}^{1(2)}\left[B_{i i}^{\alpha}(k) \cos \left(\omega_{\alpha}(k) t\right)+D_{i i}^{\alpha}(k) \sin \left(\omega_{\alpha}(k) t\right)\right] e^{-\sigma_{\alpha}(k) t}, i=n, J^{L}
$$
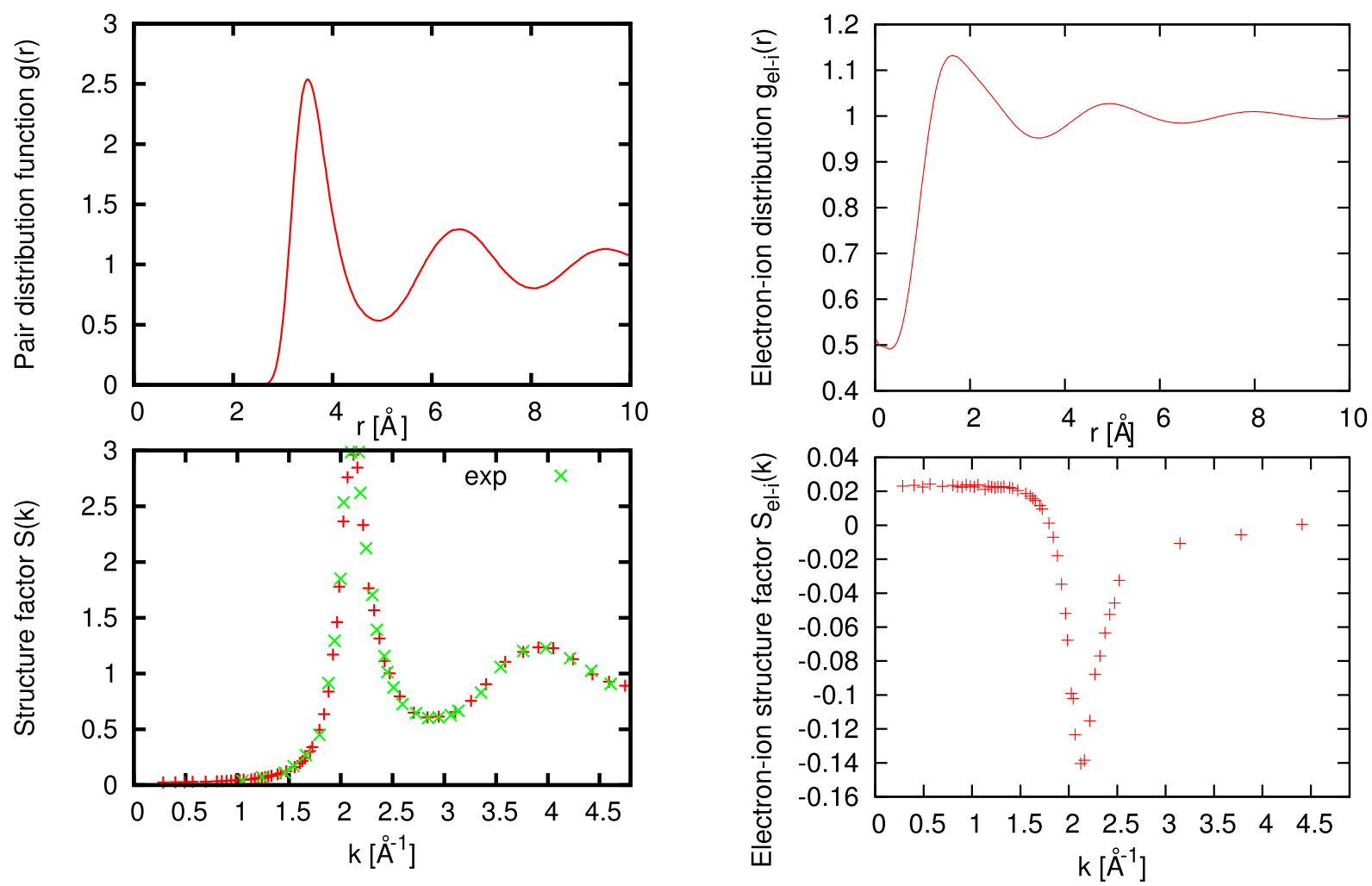

FIG. 1. Structural properties of liquid $\mathrm{Na}$ at $393 \mathrm{~K}$ and ambient pressure: pair distribution function $g(r)$ and structure factor $S(k)=\left\langle n_{-k} n_{k}\right\rangle$, electron-ion distribution function $g_{e l-i}(r)$, and electron-ion structure factor $S_{e l-i}(k)$ obtained from AIMD simulations. By cross symbols is shown the experimental structure factor $\mathrm{S}(\mathrm{k})$ for liquid $\mathrm{Na}$ at $376 \mathrm{~K}$ taken from Ref. 40 and renormalized for the actual density in AIMD at $393 \mathrm{~K}$. 

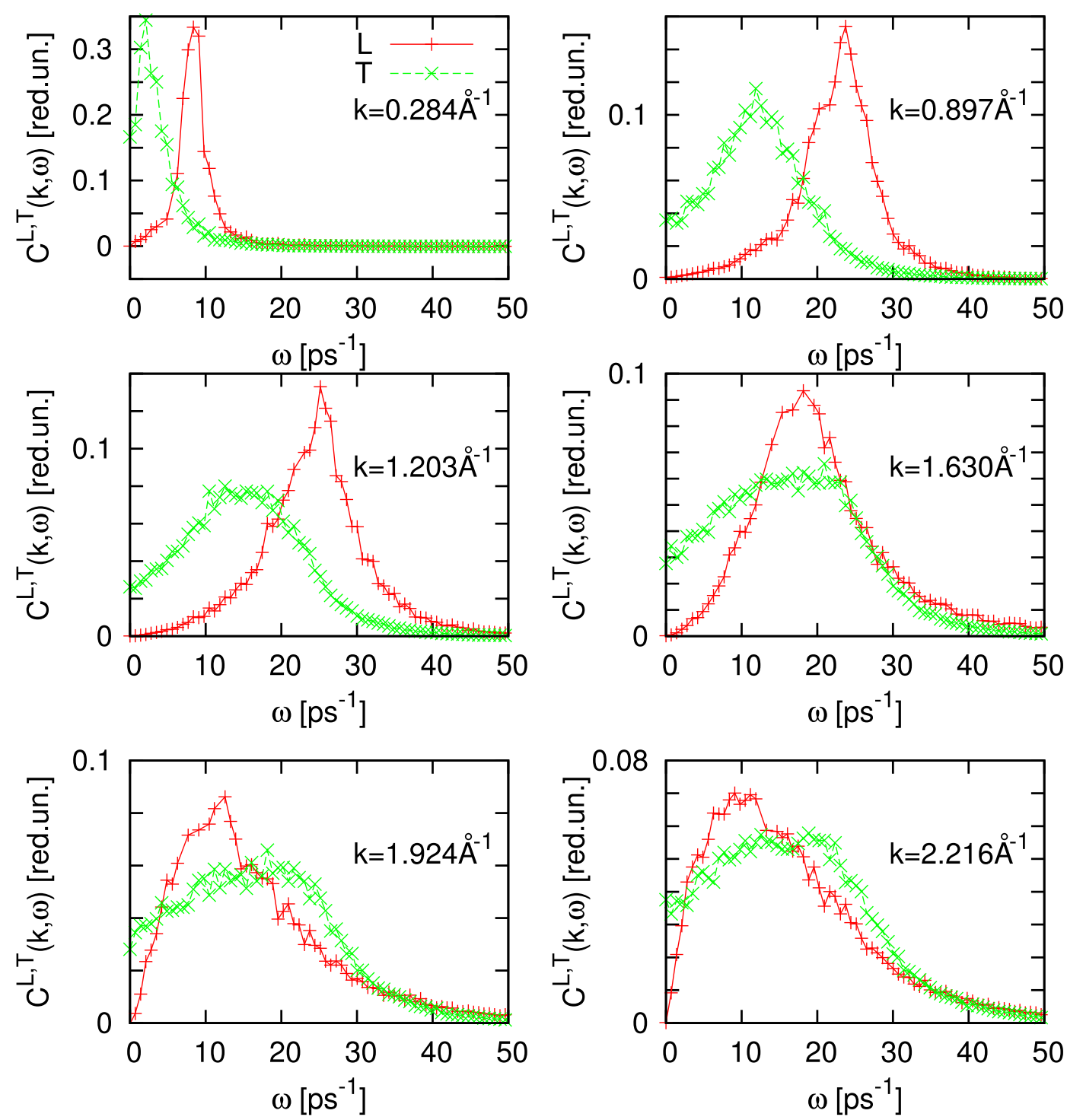

FIG. 2. Longitudinal (L) and transverse (T) current spectral functions $C^{L / T}(k, \omega)$ for liquid Na at six wave numbers.

Note that depending on the region of wave numbers, this model allows to have one type of propagating excitations and three relaxing modes (three Lorentzian contributions to the central peak of dynamic structure factor) or two types of propagating modes plus a single relaxing mode (one Lorentzian for the central peak of $S(k, \omega)$ ). The advantage of this fitting approach based on the GCM methodology is twofold: (i) the level of fulfilled exact sum rules for (9) for the density-density time correlation functions is five (constrained short-time behaviour up to the fourth time derivative at the origin) and five constraints for the longitudinal current-current time correlation functions (constrained shorttime behaviour up to the their second time derivative at the origin plus two integral sum rules-see Ref. 28); (ii) there is an explicit correspondence between the set of dynamic variables and types of non-hydrodynamic collective modes that can be obtained from the GCM generalized hydrodynamic approach as it was shown in examples of non-hydrodynamic structural relaxation, shear- and heat-waves, and optic-like excitations in many-component mixtures. ${ }^{7,31-33}$ Hence, the
GCM methodology provides a theoretical basis for the nonhydrodynamic collective excitations, while a simple fitting approach with two DHOs leads only to a guess what kind of excitations can correspond to the non-acoustic DHO.

\section{RESULTS AND DISCUSSION}

The static structure of liquid metal is usually analysed via the ionic pair distribution functions $g(r)$ and static structure factor $S(k)$. In Fig. 1, we show $g(r)$ and $S(k)$ calculated from $a b$ initio MD simulations, which are in nice agreement with previous classical simulations performed with two-body effective potentials for liquid $\mathrm{Na}^{40}$ We show by cross symbols the experimental $\mathrm{S}(\mathrm{k})$ at $376 \mathrm{~K}$ taken from Ref. 40 and renormalized due to the density difference with the actual simulations at $393 \mathrm{~K}$. Also in Fig. 1, the electronion distribution function $g_{e l-i}(r)$ and corresponding static structure factor are shown. For the purpose of further analysis of collective dynamics, we note that the main peak position of $S(k)$ is located at $k=2.03 \AA^{-1}$ and this location usually defines 


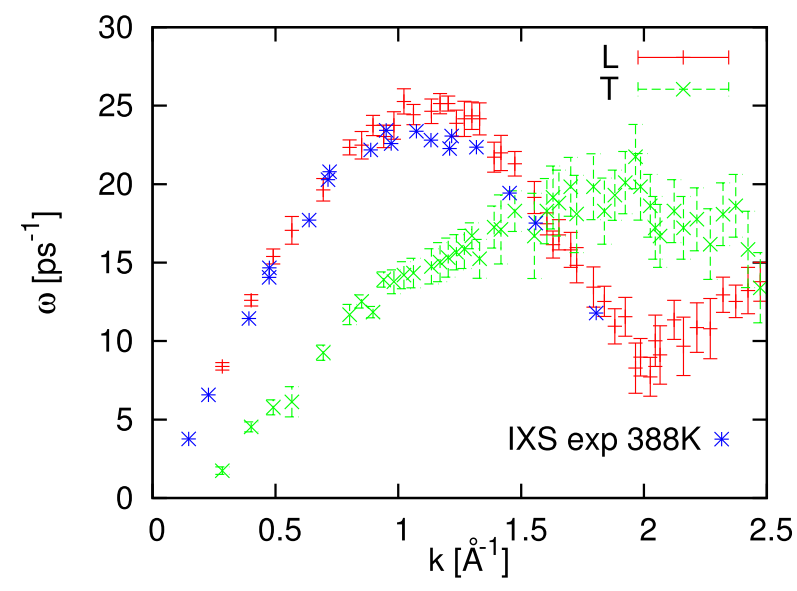

FIG. 3. Dispersion of collective excitations estimated from the peak positions of $\mathrm{L}$ (red symbols with error bars) and $\mathrm{T}$ (green symbols with error bars) current spectral functions. The experimental dispersion of longitudinal excitations obtained from IXS experiments on liquid $\mathrm{Na}$ at $388 \mathrm{~K}^{30}$ is shown by star symbols.

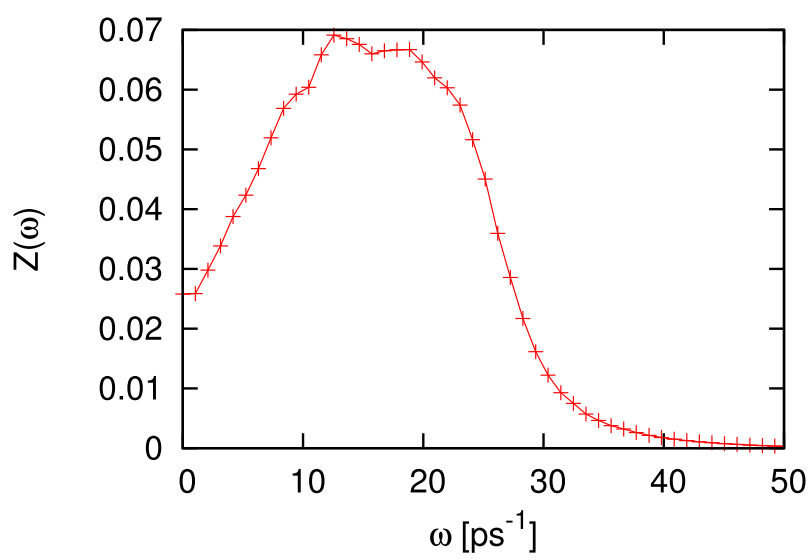

FIG. 4. Fourier-transformed velocity autocorrelation function, which outside the low-frequency region contains vibrational density of states for liquid $\mathrm{Na}$.

the region of de Gennes slowing down of density fluctuations. The electron-ion structure factor obtained directly as static averages $S_{e l-i}(k)=\left\langle n_{-k}^{e l} n_{k}^{i}\right\rangle$ has the pronounced minimum right at the location of the main peak of ionic $S(k)$ that is

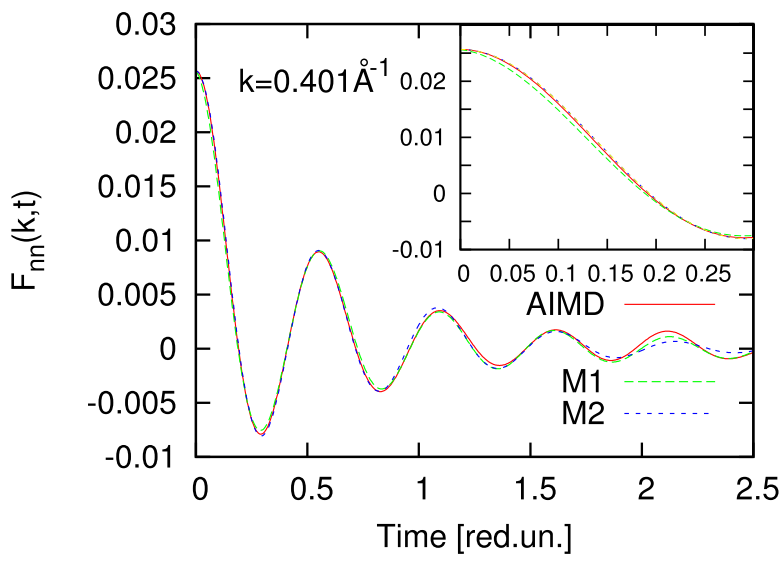

typical for simple liquid metals ${ }^{34,35}$ and can be easily obtained from linear response theory ${ }^{36}$ or other approaches. ${ }^{37-39}$

In Fig. 2, we show the frequency dependence of longitudinal and transverse current spectral functions $C^{L / T}(k, \omega)$ in a wide range of wave numbers. The collection of peak locations of $C^{L / T}(k, \omega)$ at different wave numbers is usually associated with the dispersion of $\mathrm{L}$ and $\mathrm{T}$ collective excitations, although such a methodology is not very precise and usually has large error bars for wave numbers outside the first pseudo-Brillouin zone. The peaks for the transverse spectral functions $C^{T}(k, \omega)$ are usually less pronounced because for the transverse case does not apply the condition for the longitudinal spectral function $C^{L}(k, \omega=0) \equiv 0$, which is the consequence of the continuity equation. Instead, the zero-frequency value of $C^{T}(k, \omega=0)=k_{B} T / m \rho / \eta(k) k^{2}$ is inversely proportional to the wavenumber-dependent shear viscosity of the liquid. We observed even for the smallest wave number in our simulations the peak of the transverse spectral function that gave evidence of existing shear waves even at $k=0.284 \AA^{-1}$. The dispersion of $\mathrm{L}$ and $\mathrm{T}$ collective excitations obtained directly from the peak positions of the $\mathrm{L} / \mathrm{T}$ current spectral functions is shown in Fig. 3. We were able to find in the literature the IXS data for peak positions of $C^{L}(k, \omega)$ at temperature $388 \mathrm{~K}$ (shown in Fig. 3 by star symbols). One can see that there is good agreement between our peak positions obtained from simulation and the IXS experiments. The macroscopic speed of sound in liquid cannot be estimated directly from the lowest $k$-point reached in simulations unless this $k$-value is pretty small and is located within the hydrodynamic region. Usually the smallest $k$-point in $a b$ initio simulations is outside the hydrodynamic regime and the apparent speed of sound $\omega(k) / k$ is higher than the hydrodynamic one because of the viscoelastic transition from the hydrodynamic to the high-frequency sound. The estimation of the macroscopic adiabatic speed of sound will be given below where the results of the thermo-viscoelastic analysis of longitudinal dynamics are reported. Here we have to mention that the transverse branch tends to be zero in the long-wavelength region at approximately $0.2 \AA^{-1}$ that will correspond to the width of the propagation gap for long-wavelength transverse collective excitations. The information on the density of vibrational states can be obtained from the Fourier-transformed velocity

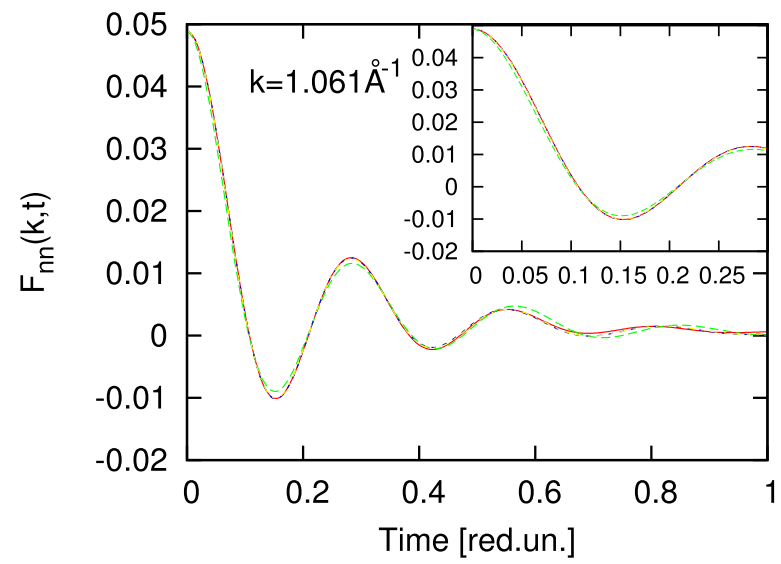

FIG. 5. Ab initio derived density-density time correlation functions (solid red line) for two wave numbers with their fit replicas using the M1 (dashed green line) and M2 (dotted blue line) fit models. The insets show the difference in quality of the short-time fit for the two models. 

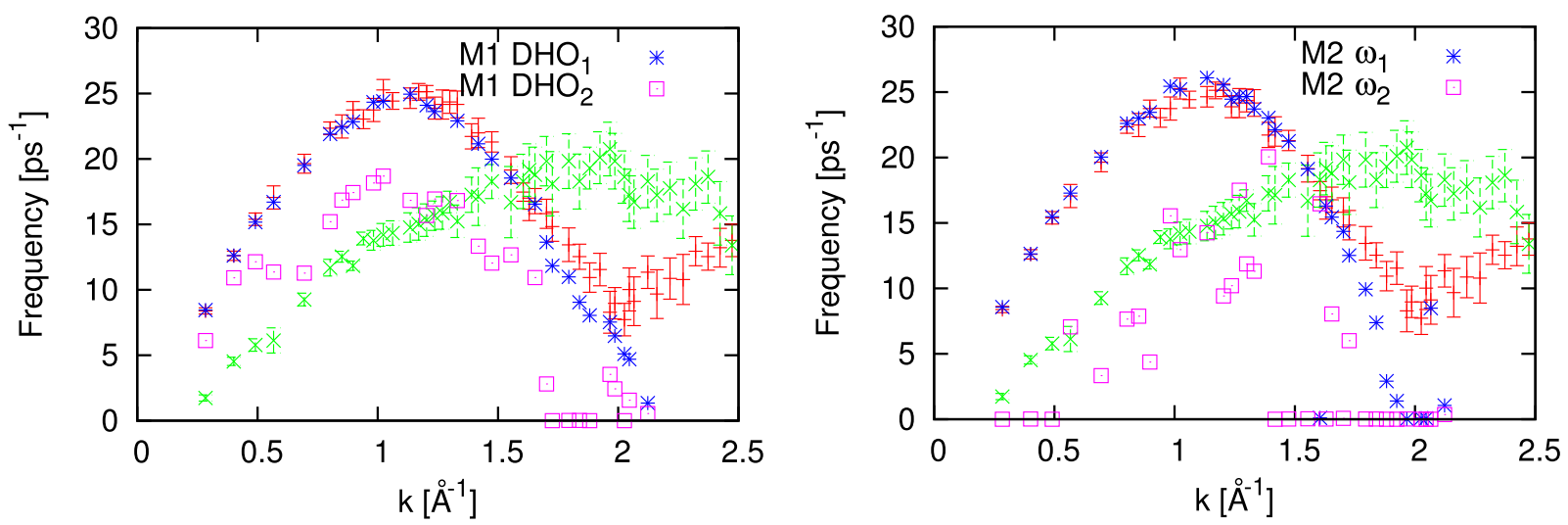

FIG. 6. Frequencies of DHOs, obtained from a fit to the ab initio derived density-density time correlation functions using models M1 (a) and M2 (b).

autocorrelation function $\tilde{Z}(\omega)$ (see Fig. 4). The zero-frequency value $\tilde{Z}(\omega=0)$ is defined by the diffusivity, but outside the low-frequency region, the smeared-out maximum of $\tilde{Z}(\omega)$ usually corresponds to the region of frequencies where the dispersion curves are slowly changing their frequency with wave number. In Fig. 4, we see that the smeared-out peaks of $\tilde{Z}(\omega)$ are located in the frequency range $10-25 \mathrm{ps}^{-1}$ that, namely, corresponds to the frequencies of short-wavelength longitudinal and transverse excitations in Fig. 3.

To analyse the collective dynamics, we start from two models, M1 and M2, which contain contributions from two types of collective excitations and were constrained by different level of exact sum rules: M1 model was constrained just by the lowest sum rule (equivalent of the constraint on zeroth frequency moment), while M2 model was by three sum

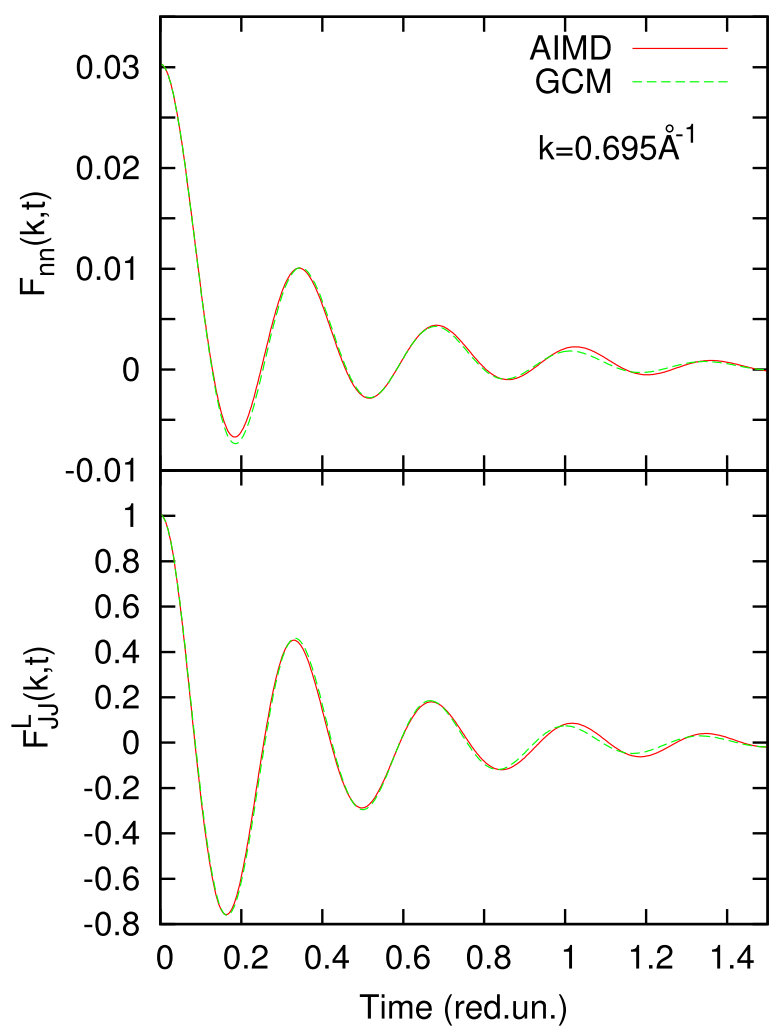

rules (up to the second frequency moment of $S(k, \omega)$ ). The quality of these two fits is seen from Fig. 5 for two wave numbers. As expected, the model M2 describes better the short-time behaviour of the density-density time correlation functions, while model M1 is not able to recover the zero slope at the origin of the AIMD-derived time correlation functions although in general their oscillating behaviour is described quite well by both models M1 and M2.

The dispersions of frequencies that correspond to the locations of the two DHOs for models M1 and M2 are shown in Fig. 6. The main contribution comes from the DHO with the frequency of the L collective excitations and nicely corresponds in both cases to the peak positions of the AIMD-calculated $C^{L}(k, \omega)$. However, the frequencies of the second DHO obtained from the M1 and M2 fits do not show

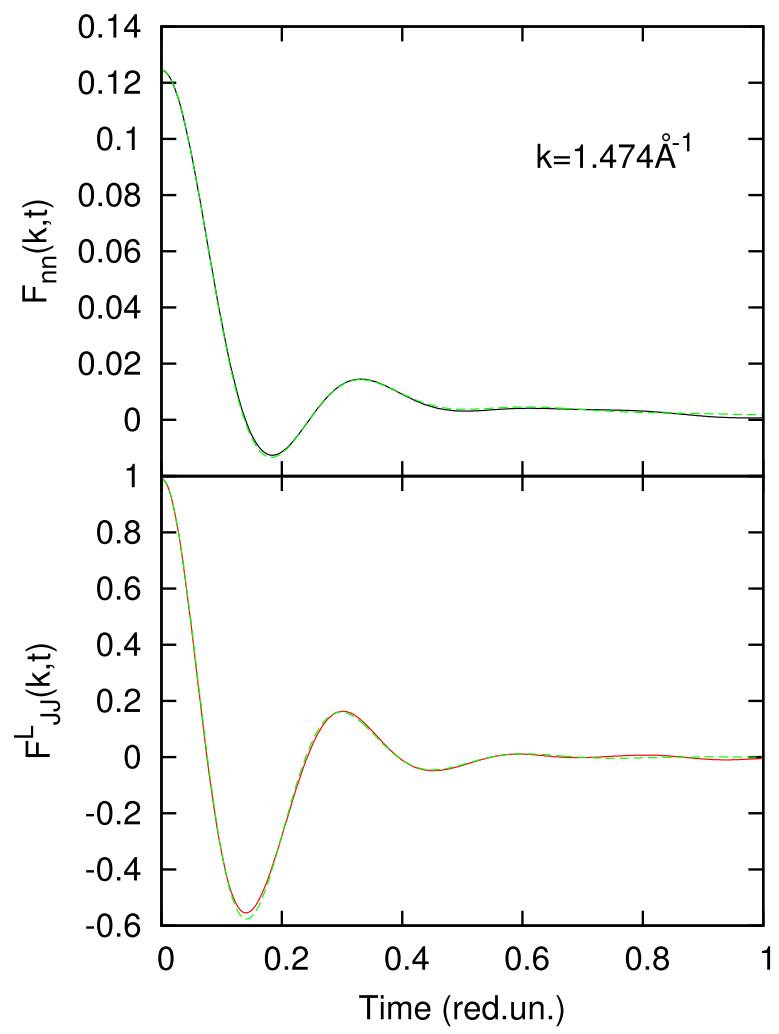

FIG. 7. Quality of the GCM replicas obtained within the thermo-viscoelastic dynamic model $\mathbf{A}^{(5)}$ in comparison with $a b$ initio derived density-density $F_{n n}(k, t)$ and longitudinal current-current $F_{J J}^{L}(k, t)$ time correlation functions at two wave numbers. 
a reasonable agreement with the dispersion of T-excitations obtained from $C^{T}(k, \omega)$. Moreover, both models M1 and M2 lead to practically vanishing frequencies of the second excitation in the region $k>1.7 \AA^{-1}$, while the AIMD gave evidence of smeared-out peaks of $C^{T}(k, \omega)$ for those wave numbers in the frequency range $17-20 \mathrm{ps}^{-1}$. Also, in the region of small wave numbers, the model M1 gave the frequencies of the non-acoustic DHO which were too high for the Texcitations, while the application of the additional sum rules in the model M2 led to zero-frequencies (i.e., non-propagating nature) of the non-acoustic excitation. These results do not allow to identify the non-acoustic DHO (in the ansatz with the two DHOs contribution to the density fluctuations) as the one connected with collective T-excitations.

The third fitting model, which is the thermo-viscoelastic dynamic model (7) for longitudinal dynamics of simple liquids provides the higher number of the exact sum rules fulfilled (up to the fourth frequency moment of $S(k, \omega)$ ). The quality of the simultaneous fit to the density-density and longitudinal current-current time correlation functions for two wave numbers is shown in Fig. 7. Hence providing nice recovering of the AIMD-derived density-density and longitudinal current-current functions, the model permits to estimate the origin of the collective modes responsible for non-acoustic contributions to the time correlation functions.

The dispersion of both branches of collective excitations that were obtained within the thermo-viscoelastic model is shown in Fig. 8. Up to the wave number $\sim 1.8 \AA^{-1}$, we were able to get very nice agreement with the peak positions of $C^{L}(k, \omega)$ (cross red symbols with error bars in Fig. 8). The macroscopic speed of sound was estimated from the $a b$ initio simulations from an extrapolation towards $k \rightarrow 0$ of the dependence $f(k)=\sqrt{\gamma(k) / S(k)}$, where $\gamma(k)$ is the wavenumber-dependent ratio of specific heats and $S(k)$ is the structure factor as it was suggested in Ref. 28. The macroscopic adiabatic speed of sound is then $c_{s}=f(k \rightarrow 0) \cdot \sqrt{k_{B} T / m}$ and for liquid $\mathrm{Na}$ at $393 \mathrm{~K}$ we obtained its value to be $2450.5 \mathrm{~m} / \mathrm{s}$, which is within 3 percent deviation from the experimental value of the adiabatic speed of sound $2519.1 \mathrm{~m} / \mathrm{s} .{ }^{41}$ The knowledge of the adiabatic

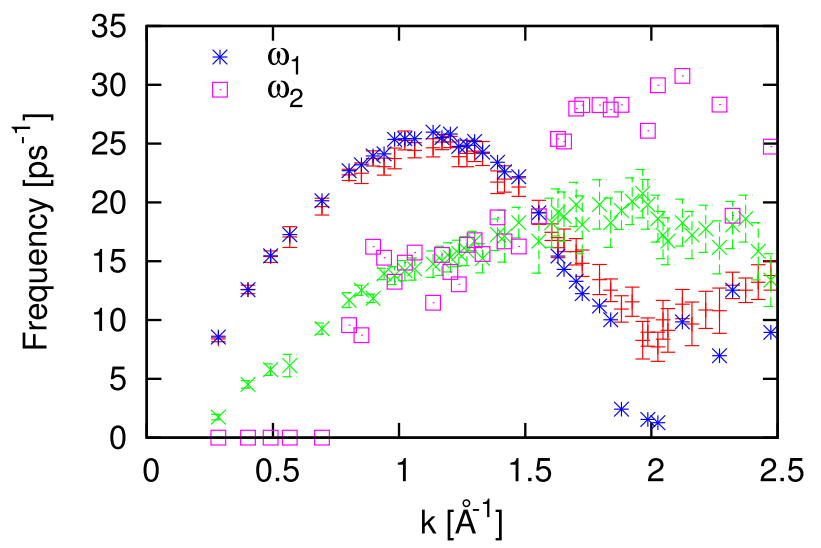

FIG. 8. Dispersion of propagating eigenvalues obtained from the GCM five-variable thermo-viscoelastic dynamic model of longitudinal dynamics in comparison with the peak positions of the $\mathrm{L}$ (red plus symbols with error bars) and $\mathrm{T}$ (green cross symbols with error bars) current spectral functions $C^{L / T}(k, \omega)$. speed of sound allows one to estimate from the obtained dispersion of L-excitations the positive sound dispersion in liquid $\mathrm{Na}$ of the order $16 \%-18 \%$, which is typical for liquid metals. From Fig. 8, one can see that the second branch of propagating excitations starts to contribute with non-zero frequencies only for wave numbers larger than $k \approx 0.75 \AA^{-1}$. Surprisingly, in the region of wave numbers up to $1.5 \AA^{-1}$, the second branch has nearly the same frequencies as the dispersion of T-excitations obtained from the peak positions of $C^{T}(k, \omega)$; however, for higher wave numbers, the second branch gets to higher frequencies than the T-excitations.

The thermo-viscoelastic dynamic model allows for the only non-acoustic type of propagating excitations which are the short-wavelength heat waves. In Ref. 32, these collective excitations were studied for the case of collective dynamics in liquid $\mathrm{Pb}$, and a simplified two-variable model of heat fluctuations was solved analytically in order to predict the existence of propagation gap for heat waves in liquids. ${ }^{7}$ Note that heat waves were known to exist in liquids for a long time. ${ }^{4}$ However, these collective modes were practically ignored in the models for density fluctuations in liquids, except the GCM approach. Very recently the contributions from the heat waves were discussed in the analysis of the density dependence of positive sound dispersion in supercritical fluids. ${ }^{8}$ In the case of liquid $\mathrm{Na}$, the short-wavelength heat waves are obtained in our approach as the eigenmodes of the generalized Langevin equation in matrix form (a system of five balance equations for dynamic variables $\left.\mathbf{A}^{(5)}(k, t)(7)\right)$ and their coupling with density fluctuations are allowed in contrast to the local coupling between the longitudinal and transverse degrees of freedom, cross-correlation between which in non-molecular one-component liquids is zero.

\section{CONCLUSION}

We performed $a b$ initio molecular dynamics simulations for liquid $\mathrm{Na}$ at $393 \mathrm{~K}$ and ambient pressure with a purpose of analysis of the obtained time correlation functions by different fitting models in order to check whether the transverse collective excitations can contribute to the longitudinal dynamics in a simple liquid metal. We applied three fitting models with different level of exact sum rules fulfilled. Two models were using a combination of two inelastic spectral shapes supposedly attributed to $\mathrm{L}$ and $\mathrm{T}$ excitations constrained by one and three sum rules. We found that the location of the low-frequency DHO from the fit does not correspond to the dispersion of the T-excitations obtained directly from the peak positions of AIMD-derived transverse current spectral functions $C^{T}(k, \omega)$. Both M1 and M2 fitting models were unable to recover the high-frequency dispersion of T-excitations for wave numbers $k>1.5 \AA^{-1}$.

The third fitting model which was based on the thermo-viscoelastic model of longitudinal dynamics of onecomponent liquid was able to recover very nicely and simultaneously the AIMD density-density and longitudinal current-current time correlation functions in a wide range of wave numbers. We stress that attributing the low-frequency DHO to the transverse excitations in the fit to experimental $S(k, \omega)$ or to the calculated in simulations density-density 
time correlation function does not have any theoretical basis and is solely suggested from an approximate location of the DHO frequency and the simulation-obtained dispersion of T-excitations in a rather narrow region of wave numbers. Our analysis with the thermo-viscoelastic dynamic model supports the possibility of a small contribution from non-hydrodynamic excitations to the density-density or longitudinal currentcurrent time-dependent correlations. However in this scheme, the non-hydrodynamic propagating excitations have thermal origin and correspond to heat waves which along with the standard thermal relaxation take part in heat transfer on nanoscale in liquids. The heat waves are supported by extended hydrodynamic theory and their local coupling with density fluctuations is not prohibited by symmetry reasons as it is with the transverse excitations. Hence, we suggest that the analysis of scattering experiments in liquid $\mathrm{Ga}^{11}$ and $\mathrm{Na}^{14}$ could indicate the presence of a signal from heat waves rather from the transverse excitations.

\section{ACKNOWLEDGMENTS}

The calculations have been performed using the ab initio total-energy and molecular dynamics program VASP (Vienna $a b$ initio simulation program) developed at the Institute für Materialphysik of the Universität Wien. ${ }^{42-44}$

${ }^{1}$ J.-P. Boon and S. Yip, Molecular Hydrodynamics (McGraw-Hill, New York, 1980).

${ }^{2}$ J.-P. Hansen and I. R. McDonald, Theory of Simple Liquids, 3rd ed. (Academic Press, London, 2006).

${ }^{3}$ T. Scopigno and G. Ruocco, Rev. Mod. Phys. 77, 881 (2005).

${ }^{4}$ D. D. Joseph and L. Preziosi, Rev. Mod. Phys. 61, 41 (1989).

${ }^{5}$ T. Bryk and I. Mryglod, Condens. Matter Phys. 11, 139 (2008).

${ }^{6}$ T. Bryk and I. Mryglod, J. Phys.: Condens. Matter 13, 1343 (2001).

${ }^{7}$ T. Bryk, Eur. Phys. J. Spec. Top. 196, 65 (2011).

${ }^{8}$ F. A. Gorelli, T. Bryk, M. Krisch, G. Ruocco, M. Santoro, and T. Scopigno, Sci. Rep. 3, 1203 (2013)

${ }^{9}$ M. Sampoli, G. Ruocco, and F. Sette, Phys. Rev. Lett. 79, 1678 (1997).

${ }^{10}$ A. Cimatoribus, S. Saccanim, F. Bencivenga, A. Gessini, M. G. Izzo, and C. Masciovecchio, New J. Phys. 12, 053008 (2010).

${ }^{11}$ S. Hosokawa, M. Inui, Y. Kajihara, K. Matsuda, T. Ichitsubo, W.-C. Pilgrim, H. Sinn, L. E. González, D. J. González, S. Tsutsui, and A. Q. R. Baron, Phys. Rev. Lett. 102, 105502 (2009).
${ }^{12}$ V. M. Giordano and G. Monaco, Phys. Rev. B 84, 052201 (2011).

${ }^{13}$ S. Hosokawa, S. Munejiri, M. Inui, Y. Kajihara, W.-C. Pilgrim, Y. Ohmasa, S. Tsutsui, A. Q. R. Baron, F. Shimojo, and K. Hoshino, J. Phys.: Condens. Matter 25, 112101 (2013).

${ }^{14}$ V. M. Giordano and G. Monaco, Proc. Natl. Acad. Sci. U. S. A. 107, 21985 (2010).

${ }^{15}$ S. Hosokawa, M. Inui, Y. Kajihara, S. Tsutsui, and A. Q. R. Baron, J. Phys.: Condens. Matter 27, 194104 (2015).

${ }^{16}$ M. Zanatta, F. Sacchetti, E. Guarini, A. Orecchini, A. Paciaroni, L. Sani, and C. Petrillo, Phys. Rev. Lett. 114, 187801 (2015).

${ }^{17}$ S. Munejiri, F. Shimojo, and K. Hoshino, Phys. Rev. B 86, 104202 (2012).

${ }^{18}$ T. Bryk, G. Ruocco, T. Scopigno, and A. P. Seitsonen, J. Chem. Phys. 143, 110204 (2015).

${ }^{19}$ M. Marqués, L. E. González, and D. J. González, Phys. Rev. B 92, 134203 (2015).

${ }^{20}$ P. Verkerk, J. Phys.: Condens. Matter 13, 7775 (2001).

${ }^{21}$ C. Cohen, J. W. H. Sutherland, and J. M. Deutch, Phys. Chem. Liq. 2, 213 (1971).

${ }^{22}$ B. Dorner, in Introduction to Neutron Scattering. Lecture Notes of the Introductory Course, edited by A. Furrer (Paul Scherrer Institut - Villigen, Switzerland, 1996).

${ }^{23}$ P. E. Blöchl, Phys. Rev. B 50, 17953 (1994).

${ }^{24}$ G. Kresse and D. Joubert, Phys. Rev. B 59, 1758 (1999).

${ }^{25}$ N. Jakse and A. Pasturel, Sci. Rep. 3, 3135 (2013).

${ }^{26}$ N. Jakse and A. Pasturel, Condens. Matter Phys. 18, 43603 (2015).

${ }^{27}$ I. M. Mryglod, I. P. Omelyan, and M. V. Tokarchuk, Mol. Phys. 84, 235 (1995).

${ }^{28}$ T. Bryk and G. Ruocco, Mol. Phys. 111, 3457 (2013).

${ }^{29}$ T. Bryk and I. Mryglod, Phys. Rev. E 64, 032202 (2001).

${ }^{30}$ W.-C. Pilgrim, S. Hosokawa, S. Saggau, H. Sinn, and E. Burkel, J. NonCryst. Solids 250-252, 96 (1999).

${ }^{31}$ T. Bryk and I. Mryglod, J. Phys.: Condens. Matter 12, 6063 (2000).

${ }^{32}$ T. Bryk and I. Mryglod, Phys. Rev. E 63, 051202 (2001).

${ }^{33}$ T. Bryk and A. B. Belonoshko, Phys. Rev. B 86, 024202 (2012).

${ }^{34}$ G. A. de Wijs, G. Pastore, A. Selloni, and W. van der Lugt, Phys. Rev. Lett. 75, 4480 (1995).

${ }^{35}$ J.-F. Wax, N. Jakse, and J.-L. Bretonnet, Phys. Rev. B 55, 12099 (1997).

${ }^{36}$ T. Bryk, I. Klevets, G. Ruocco, T. Scopigno, and A. P. Seitsonen, Phys. Rev. B 90, 014202 (2014).

${ }^{37}$ S. K. Lai, K. Horii, and M. Iwamatsu, Phys. Rev. E 58, 2227 (1998).

${ }^{38}$ J. Chihara and G. Kahl, Phys. Rev. B 58, 5314 (1998).

${ }^{39}$ I. Klevets and T. Bryk, J. Chem. Phys. 141, 214504 (2014).

${ }^{40}$ U. Balucani, A. Torcini, and R. Vallauri, Phys. Rev. B 47, 3011 (1993).

${ }^{41}$ R. T. Beyer, in Handbook of Thermodynamic and Transport Properties of Alkali Metals, edited by R. W. Ohse (Blackwell Scientific Publications, Oxford, 1985), p. 525.

${ }^{42}$ G. Kresse and J. Hafner, Phys. Rev. B 47, 558 (1993); ibid. 49, 14251 (1994).

${ }^{43}$ G. Kresse and J. Furthmüller, Comput. Mater. Sci. 6, 15 (1996).

${ }^{44}$ G. Kresse and J. Furthmüller, Phys. Rev. B 54, 11169 (1996). 